\title{
Printed subthreshold organic transistors operating at high gain and ultralow power
}

\author{
Chen Jiang ${ }^{1,2}$, Hyung Woo Choi ${ }^{1}$, Xiang Cheng ${ }^{1,3}$, Hanbin Ma ${ }^{1,4,5}$, David Hasko ${ }^{1}$, Arokia \\ Nathan ${ }^{1,3,5 *}$
}

\begin{abstract}
Affiliations:
${ }^{1}$ Electrical Engineering Division, Department of Engineering, University of Cambridge, $9 \mathrm{JJ}$ Thomson Avenue, Cambridge CB3 OFA, United Kingdom.

${ }^{2}$ Department of Clinical Neurosciences, University of Cambridge, Cambridge Biomedical Campus, CB2 0QQ, United Kingdom.

${ }^{3}$ Cambridge Touch Technologies Ltd, 154 Cambridge Science Park, Cambridge CB4 0GN, United Kingdom.

${ }^{4}$ Suzhou Institute of Biomedical Engineering and Technology, Chinese Academy of Sciences, Suzhou, 215163, China.

${ }^{5}$ Acxel Tech Ltd, 184 Cambridge Science Park, Cambridge CB4 0GA, United Kingdom.

*Correspondence to: an299@ cam.ac.uk
\end{abstract}

Abstract (<125 words): Overcoming the trade-offs between power consumption, fabrication-cost and signal-amplification has been a long-standing question for wearable electronics. We report a high-gain, fully inkjet-printed Schottky-barrier organic thin-film transistor amplifier circuit. The transistor signal amplification efficiency is 38.2 siemens per ampere, which is near the theoretical thermionic limit and an ultralow power consumption of $<1$ nanowatt. The use of a Schottky-barrier for the source gave the transistor geometry-independent electrical characteristics and accommodated the large dimensional-variation in inkjet-printed features. These transistors demonstrated good reliability with negligible threshold-voltage shift. We demonstrate this capability with an ultralow-power high-gain amplifier for the detection of electrophysiological signals and showed a signal-to-noise ratio $>60$ decibels and noise voltage $<0.3$ microvolt per hertz $^{1 / 2}$ at 100 hertz.

One Sentence Summary: A low cost, low power, stable and flexible transistor fabrication technology is used to make a wearable micro-volt resolution electrophysiological signal detection amplifier.

30 Main Text (<2500 words): Organic thin-film transistors (OTFTs) have driven the development in low-cost, large-area electronics, including emerging application areas (1-9) such as wearable technologies. These applications require devices that are bendable and stretchable without affecting their electrical behavior $(10,11)$. Organic semiconductors have been widely investigated for this application, but circuits usually require a large operating voltage, leading to high power consumption and unsuitability for battery powered operation (12-14). The most challenging part of wearable electronics is the sensor interface, which is an analog application requiring lowvoltage, low-power circuits with high gain, very high input impedance, low noise (15) and simple, low-cost fabrication $(16,17)$.

To meet these requirements, we use an inkjet-printed circuit technology (18) based on a subthreshold Schottky-barrier (SB) OTFT that operates near the OFF state. This approach has three 
main advantages (19). First, these transistors exhibit a steep subthreshold slope, which allows the use of a low operating voltage and leads to a high transconductance efficiency. Second, the currentvoltage $(I-V)$ characteristics are independent of the channel length for a broad range of device geometries. These characteristics are ideal for printed electronics, because the variation in the typical inkjet-printed feature size of $\sim 40 \mu \mathrm{m}$ can be as much as $10 \mu \mathrm{m}$ (fig. S12). Third, the intrinsic gain of the SB-OTFT transistor is large (e.g., > 1000) and independent of channel length and electrical bias, with a $V-I$ signal amplification efficiency approaching the theoretical limit of $q / k_{\mathrm{B}} T$, where $q$ is the elementary charge, $k_{\mathrm{B}}$ is Boltzmann's constant, and $T$ is temperature.

Defect density must be minimized within the printed structure to ensure a good Schottkybarrier contact at the source-semiconductor interface (19). The Schottky-contact energy barrier for hole injection into the organic semiconductor is established by the difference between the work function of the metal and the highest occupied molecular orbit level (HOMO) in the organic semiconductor (19-21). We used 2,7-dioctyl[1]benzothieno[3,2-b][1]benzothiophene (C8-BTBT) as the semiconductor (Fig. 1A), which exhibits fast growth $(<1 \mathrm{~min})$ of large crystals $(>50 \mu \mathrm{m})$, and has a lower HOMO level compared to pentacene or other derivatives (22) to yield a good Schottky-barrier $(>0.2 \mathrm{eV})(19,21)$. Polyvinyl cinnamate (PVC) was used as the dielectric layer to provide a smooth interface between the semiconductor and dielectric, thus minimizing carrier trapping and scattering (23). A fluoropolymer encapsulation layer (CYTOP) protected the device from environmental effects. Silver was used for the metal parts. All of these materials were formulated as inks with good jetting properties (Fig. S1), and all of the fabrication steps for the individual SB-OTFT transistors and amplifier circuits reported here were carried out using a single inkjet printer tool.

The SB-OTFT demonstrated a near-zero threshold voltage $\left(V_{\mathrm{T}}=-0.01 \mathrm{~V}\right.$; Fig. 1B) along with an ultrasteep subthreshold slope of $S S=60.2 \mathrm{mV} /$ decade (Fig. 1C) that approached the theoretical thermionic limit (20):

$$
S S_{\text {theoretical }}=\ln (10) v_{t h}=59.6 \mathrm{mV} / \text { decade }(\text { at } T=300 \mathrm{~K}),
$$

where $v_{\text {th }}=k_{\mathrm{B}} T / q$ is thermal voltage. In addition, this steep $S S$ is repeatable (Fig. S3). The small $V_{\mathrm{T}}$ and steep $S S$ were resulted from the low trap density (20):

$$
V_{T}=V_{T, \text { theoretical }}+\frac{Q_{t}}{C_{i}}
$$

and

$$
S S=S S_{\text {theoretical }}\left(1+\frac{q^{2} D_{t}}{C_{i}}\right),
$$

where $Q_{\mathrm{t}}$ is the trap carrier density, coulomb per $\mathrm{cm}^{2}, D_{\mathrm{t}}$ is the defect trap density, per $\mathrm{eV}$ and $\mathrm{cm}^{2}$, and $C_{\mathrm{i}}$ is the gate insulator capacitance, farad per $\mathrm{cm}^{2} . Q_{\mathrm{t}}$ and $D_{\mathrm{t}}$ can be affected by defects in the semiconductor bulk (e.g., grain boundaries and stacking faults) and at the semiconductor/dielectric interface (e.g., interface roughness and atomic species/vacancies on dangling bonds). The relatively large semiconductor crystals in the TFT channel $(>50 \mu \mathrm{m}$, providing good coverage over the channel, Fig. S2E) significantly reduce grain boundaries and stacking faults, as compared to the amorphous or micro-polycrystalline phases. The printed polymer dielectric layer was free of dangling bonds and provided a smooth semiconductor/dielectric interface (with roughness of 2.1 
$\AA$, Fig. S2C). This was comparable to the roughness of the silicon/silicon dioxide in state-of-theart CMOS technologies. Thus, reducing the defect density to a very low level gives the best values for $V_{\mathrm{T}}$ and $S S$; furthermore, the variation in these values between devices was much less than for other vacuum-deposition-based TFT technologies (Fig. 1, D and E, Table S1). Note that TFTs with a large $C_{\mathrm{i}}$ are effective in reducing $V_{\mathrm{T}}$ and $S S$ (24), but lead to higher operating current. While this boosts the switching speed in logic circuits, it does not benefit the low-power, low frequency operation of analog sensor interfaces.

We investigated the nature of the defect density and of the Schottky barrier through the density of states (DOS), see Fig. 2A and the effective Schottky-barrier height ( $\Phi_{\text {eff }}$ ). These results suggest that the DOS comprises a small and constant background of deep states $\left(g_{\text {deep }}=6.59 \times 10^{14}\right.$ $\mathrm{cm}^{-3} \mathrm{eV}^{-1}$ ), a broad spectrum of delocalized states with a characteristic energy of $24.8 \mathrm{meV}$ near $v_{\text {th }}$, and a steeply rising number of localized tail states with a characteristic energy of $6.7 \mathrm{meV}$. In addition, because the DOS was dominated by extended states (following $\sqrt{E-E_{\text {HOMO }}}$ ), there was a clear mobility edge for energies above the HOMO level (i.e., $E>E_{\mathrm{HOMO}}$ ) characteristic of a small overall DOS. Because the semiconductor source potential $\left(\varphi_{\mathrm{S}}\right)$ cannot be neglected in low-voltage TFTs, this term was included in the DOS calculation (Eqs. S5 to S23).

The source-side Schottky-barrier height ( $\left.\Phi_{\text {eff }}\right)$ decreased with increasing $-V_{\mathrm{GS}}$, so that the drain current $\left(I_{\mathrm{DS}}\right)$ was modulated by the gate bias. $\Phi_{\text {eff }}$ could be extracted from temperaturedependent $I-V$ measurements (Fig. S6). In the subthreshold regime, $\Phi_{\text {eff }}=\zeta_{0} V_{\mathrm{GS}}+\Phi_{\mathrm{eff}, 0}$, where $\zeta_{0}$ is a coefficient that describes the modulation of Schottky-barrier height by $V_{\mathrm{GS}}(19)$. $\Phi_{\text {eff }}$ showed a good initial Schottky-barrier of $\sim 0.51 \mathrm{eV}$ and a high barrier-lowering factor of $\zeta_{0}=1.24$. This result suggests that charge-carrier injection was mainly by thermionic emission with smaller contributions from thermionic field emission and tunneling (see inset of Fig. 2B). Note that above a certain $V_{\mathrm{GS}}$ level (in the case shown at $-0.34 \mathrm{~V}$ ), barrier lowering saturated, and the transistor behaved ohmically in the above-threshold regime. This change occurred when the source-side depletion width reached just a few nanometers and allowed charge carriers to tunnel through the Schottky barrier (Fig. S5C) $(20,21)$. The small defect density and the presence of a good Schottky barrier in the subthreshold regime were prerequisites for a high $g_{\mathrm{m}}$ and $r_{\mathrm{o}}$.

The near-zero $V_{\mathrm{T}}$ was important for low-power operation, whereas the ultrasteep $S S$ was important for high transconductance $\left(g_{m}=\partial I_{D S} / \partial V_{G S}\right)$ and transconductance efficiency $\left(g_{m} / I_{D S}\right)$ (Eqs. S2 and S3). In addition, the SB-OTFT operation was channel-length independent with a large output resistance $\left(r_{O}=\partial V_{D S} / \partial I_{D S}\right.$, Fig. $\left.1 \mathrm{~F}\right)$, which was provided by the Schottky barrier at the source-semiconductor contact. Thus, the SB-OTFT could provide a high intrinsic gain (defined as $A_{\mathrm{i}}=g_{\mathrm{m}} r_{\mathrm{o}}$ ) (25), resulting from the high transconductance and output resistance.

Both the transconductance and output resistance had an exponential dependence with an inverse proportionality on $-V_{\mathrm{GS}}$, because of the response of SB-TFTs in the subthreshold regime (Fig. 2C) as was also the case previously with an inorganic SB-TFT (19). In comparison with other TFT technologies, the SB-OTFT transconductance and output resistance is about ten times higher at similar currents, i.e., $g_{\mathrm{m}}=3.8 \times 10^{-8}$ siemens and $r_{\mathrm{o}}=3.2 \times 10^{10} \mathrm{ohm}$ at $I_{\mathrm{DS}}=1 \mathrm{nA}(1-6,24,26$ 28). The intrinsic gain $A_{\mathrm{i}}$ was determined from the theoretical expression (19): $A_{i}=$ $\frac{S S_{\text {theoretical }}}{s S} n \exp \left(\frac{v_{\text {sat }}}{n v_{t}}\right)$, where $n$ is the ideality factor (here, $\left.n=1.6\right)$. These devices showed a high and constant value for $A_{\mathrm{i}}$ of $\sim 1100$ in the subthreshold regime (Fig. 2D), which is much larger than that of the inorganic SB-TFT and Si-MOSFET because of the ultrasteep SS. More importantly, $g_{\mathrm{m}} / I_{\mathrm{DS}}$ for the SB-OTFT was $\sim 38.2 \mathrm{~S} / \mathrm{A}$, approaching the theoretical limit for TFT technologies of 
$q / k_{\mathrm{B}} T$ (i.e., $38.7 \mathrm{~S} / \mathrm{A}$ at $T=300 \mathrm{~K}$ ). The high $g_{\mathrm{m}} / I_{\mathrm{DS}}$ (indicating a large $g_{\mathrm{m}}$ at low $I_{\mathrm{DS}}$ ) was essential for an amplifier circuit to achieve high gain at low power. The SB-OTFT reported here exhibited more efficient $V$-I signal amplification compared to the other reported devices (Fig. 2E).

The usability of inkjet-printed OTFTs is commonly limited by their short shelf life and operational instabilities $(29,30)$. However, when the transfer $\left(I_{\mathrm{D}}-V_{\mathrm{GS}}\right)$ characteristics of representative SB-OTFT were tested over a period of 3 months under ambient conditions, no appreciable changes were observed (Fig. 3A). The threshold voltage shift was $<1 \mathrm{mV}$ and the transconductance efficiency changed by $<1 \%$, thus far superior in ambient environment operation and storage than typical OTFTs where these changes are typically $>100 \mathrm{mV}$ and $>20 \%$, respectively $(30,31)$.

Similarly, the effect of electrical and illumination stress was very small (29-31). Electrical stress was applied under an ON-state condition (i.e., $V_{\mathrm{GS}}=V_{\mathrm{DS}}=-3 \mathrm{~V}$ ), in which a conducting channel was formed and charge carriers were more likely to be trapped compared to the nearly OFF-state condition. The transfer characteristics of the device before and after stress were almost identical (Fig. 3C). The threshold voltage shifted by $<30 \mathrm{mV}$ with the characteristic decay time of $\sim 10^{3} \mathrm{~s}$ and the transconductance efficiency changed by $<2 \%$ (Fig. 3D). Because of the wide bandgap of C8-BTBT (Fig. S7), the device demonstrated excellent light stability (Fig. 3E) under visible light illumination stress $\left(10 \mathrm{~mW} / \mathrm{cm}^{2}\right)$, with a photocurrent $<10$ attoampere $/ \mu \mathrm{m}$ and a threshold voltage shift within $1 \mathrm{mV}$ (Fig. 3F).

Noise ultimately limits the minimum detectable signal in any circuit, especially at the low frequencies of many electrophysiological signals $(<100 \mathrm{~Hz})$. The low-frequency noise response of SB-OTFTs showed both $1 / f$ and white noise (Fig. 3G). As expected, these noise components were proportional to the current as $I^{2}$ and $I$, respectively (Fig. S8, C and D, Eqs. S29 and 30). Thus, by operating in the subthreshold regime, the noise was reduced, giving rise to a signal-to-noise ratio (SNR) of $63 \mathrm{~dB}$ over the cut-off frequency of the TFT (Fig. 3H), which is sufficient for most lowfrequency analog applications. The flicker noise coefficient is fabrication process dependent, and the value in our devices was $1.8 \times 10^{-22} \mathrm{~V}^{2}$ farad, which is one order of magnitude lower than that found in typical amorphous Si and metal oxide based TFTs and two orders of magnitude lower than conventional OTFTs (Table S1) (32). The root-mean-square noise voltage referred to the gate $30 \sqrt{\left\langle v_{g n}^{2}\right\rangle}$ for all noise sources is $<0.3 \mu \mathrm{V} / \mathrm{Hz}^{1 / 2}$ at $100 \mathrm{~Hz}$ (Fig. 3H), which is a few orders of magnitude lower than that of other TFT technologies for the same operating current.

We integrated amplifier circuits from pairs of SB-OTFTs in a common-source configuration, i.e., a drive transistor $\mathrm{T}_{\mathrm{D}}$ and a bias transistor $\mathrm{T}_{\mathrm{B}}$ (Fig. 4A). Because of the very high $A_{\mathrm{i}}$ of the SB-TFT, the amplifier demonstrated steep $V_{\text {out }}$ characteristics and a voltage gain $\left(A_{V}=\right.$ $\partial V_{\text {out }} / \partial V_{\text {in }}$ ) of $260 \mathrm{~V} / \mathrm{V}$ at the peak (Fig. 4B). Because transistor $\mathrm{T}_{\mathrm{B}}$ operated in the subthreshold regime with a bias current $I_{\mathrm{B}}=342 \mathrm{pA}$ in the saturation regime, the power consumption was $<1$ nW (Fig. 4C). Compared to other TFT amplifiers, this high-gain amplifier enabled high-resolution $(<4 \mu \mathrm{V})$ of electrophysiological signal detection (Fig. 4D). In addition, the gain-bandwidth product was scalable by gate bias. Given a maximum electrophysiological signal frequency of $50 \mathrm{~Hz}(33)$, the SB-OTFT had a relatively large allowed bias window for analog circuit design $(0.13 \mathrm{~V})$ compared to the variation of $V_{\mathrm{T}}$.

Such an amplifier can be used to monitor human electro-oculogram (EOG) signals, which are essentially the corneo-retinal potentials ( $\left.V_{\mathrm{EOG}}\right)$ that exist across the front (positive) and back (negative) of the human eye (Fig. 4F), typically in the range from 0.2 to $\sim 1.0 \mathrm{mV}$ (34). This 
technique is useful for eye-movement tracking, particularly in improving existing technologies that are bulky, costly and require high power (35). With a biasing electrode over the eyebrow and another electrode below the lower eyelid connecting to the amplifier input (Fig. 4, A and F), the $V_{\text {in }}$ relation for the amplifier becomes

$$
V_{\text {in }}=V_{\text {bias }}+\gamma V_{E O G} \text {. }
$$

Here, $\gamma$ is a coefficient that describes the direction of eye movement. In the configuration used, $\gamma<0$ corresponds to an upward movement of the eyeball, whereas $\gamma>0$ indicates the corresponding downward movement. Therefore, the amplifier output gives an amplitude of up to $\sim 0.3 \mathrm{~V}$ (Fig. 4G, Movie S1). The amplifier is also able to track horizontal eye movement (Fig. S11). The amplified EOG signal with amplitudes $>0.2 \mathrm{~V}$ and SNR $>60 \mathrm{~dB}$ has the potential to detect subtle eye movements for a better depiction of the virtual environment, e.g., depth-of-field rendering. Tracking eye movement is important in virtual and augmented reality (35). The ultralow power consumption of SB-OTFT based circuits can potentially operate from energy acquired from microharvesters (in the order of $\mu \mathrm{J} /$ cycle) (8), although from a complete system standpoint this would require low-power versions of signal conditioning and transmission circuit stages.

\section{References and Notes:}

1. R. A. Street, Thin-film transistors. Adv. Mater. 21, 2007-2022 (2009).

2. A. Nathan et al., Flexible electronics: The next ubiquitous platform. Proc. IEEE. 100, 14861517 (2012).

3. K. Nomura et al., Room-temperature fabrication of transparent flexible thin-film transistors using amorphous oxide semiconductors. Science 300, 1269-1272 (2003).

4. J. F. Wager, Transparent electronics. Science 300, 1245-1246 (2003).

5. H. Keum et al., Epidermal Electronics. Science 333, 838-844 (2011).

6. T. Someya, Z. Bao, G. G. Malliaras, The rise of plastic bioelectronics. Nature. 540, 379-385 (2016).

7. A. Campana, T. Cramer, D. T. Simon, M. Berggren, F. Biscarini, Electrocardiographic recording with conformable organic electrochemical transistor fabricated on resorbable bioscaffold. Adv. Mater. 26, 3874-3878 (2014).

8. D. Khodagholy et al., In vivo recordings of brain activity using organic transistors. Nat. Commun. 4, 1575 (2013).

9. Y. Kim et al., A bioinspired flexible organic artificial afferent nerve. Science 360, 998-1003 (2018).

10. T. Sekitani, U. Zschieschang, H. Klauk, T. Someya, Flexible organic transistors and circuits with extreme bending stability. Nat. Mater. 9, 1015-1022 (2010).

11. J. Xu et al., Highly stretchable polymer semiconductor films through the nanoconfinement effect. Science. 355, 59-64 (2017).

12. H. Nishide, K. Oyaizu, Toward Flexible Batteries. Science 319, 737-738 (2008). 
13. P. D. Mitcheson, E. M. Yeatman, G. K. Rao, A. S. Holmes, T. C. Green, Energy harvesting from human and machine motion for wireless electronic devices. Proc. IEEE. 96, 1457-1486 (2008).

14. W. Gao et al., Fully integrated wearable sensor arrays for multiplexed in situ perspiration analysis. Nature. 529, 509-514 (2016).

15. T. Sekitani et al., Ultraflexible organic amplifier with biocompatible gel electrodes. Nat. Commun. 7, 11425 (2016).

16. H. Sirringhaus et al., High-resolution Inkjet Printing of All-Polymer Transistor Circuits. Science. 290, 2123-2126 (2000).

17. L. Feng, C. Jiang, H. Ma, X. Guo, A. Nathan, All ink-jet printed low-voltage organic fieldeffect transistors on flexible substrate. Org. Electron. 38, 186-192 (2016).

18. M. Singh, H. M. Haverinen, P. Dhagat, G. E. Jabbour, Inkjet printing-process and its applications. Adv. Mater. 22, 673-685 (2010).

19. S. Lee, A. Nathan, Subthreshold Schottky-barrier thin-film transistors with ultralow power and high intrinsic gain. Science 354, 302-304 (2016).

20. S. M. Sze, Physics of Semiconductor Devices (Wiley, Hoboken, NJ, USA, 2nd ed., 1981).

21. Y. Xu, H. Sun, Y.-Y. Noh, Schottky Barrier in Organic Transistors. IEEE Trans. Electron Devices. 64, 1932-1943 (2017).

22. M. Kano, T. Minari, K. Tsukagoshi, Improvement of subthreshold current transport by contact interface modification in p-type organic field-effect transistors. Appl. Phys. Lett. 94 (2009).

23. C. Jiang, H. Ma, D. G. Hasko, X. Guo, A. Nathan, A Lewis-Acid Monopolar Gate Dielectric for All-Inkjet-Printed Highly Bias-Stress Stable Organic Transistors. Adv. Electron. Mater. 3 (2017).

24. H. Klauk, U. Zschieschang, J. Pflaum, M. Halik, Ultralow-power organic complementary circuits. Nature. 445, 745-8 (2007).

25. B. Razavi, Design of Analog CMOS Integrated Circuits (McGraw-Hill, NY, 2001).

26. M. J. Powell, The Physics of Amorphous-Silicon Thin-Film Transistors. IEEE Trans. Electron Devices. 36, 2753-2763 (1989).

27. S. D. Brotherton, Polycrystalline silicon thin film transistors. Semicond. Sci. Technol. 10, 721-738 (1995).

28. R. Martins et al., Complementary metal oxide semiconductor technology with and on paper. Adv. Mater. 23, 4491-4496 (2011).

29. W. H. Lee, H. H. Choi, D. H. Kim, K. Cho, Microstructure Dependent Bias Stability of Organic Transistors. Adv. Mater. 26, 1660-1680 (2014).

30. H. Sirringhaus, Reliability of organic field-effect transistors. Adv. Mater. 21, 3859-3873 (2009).

31. X. Jia, C. Fuentes-Hernandez, C.-Y. Wang, Y. Park, B. Kippelen, Stable organic thin-film transistors. Sci. Adv. 4 (2018), doi:10.1126/sciadv.aao1705. 
32. T. Moy et al., An EEG Acquisition and Biomarker-Extraction System Using Low-NoiseAmplifier and Compressive-Sensing Circuits Based on Flexible, Thin-Film Electronics. IEEE J. Solid-State Circuits. 52, 309-321 (2017).

33. D. Mantini, M. G. Perrucci, C. Del Gratta, G. L. Romani, M. Corbetta, Electrophysiological signatures of resting state networks in the human brain. Proc. Natl. Acad. Sci. 104, 13170-5 (2007).

34. M. Brown et al., ISCEV Standard for Clinical Electro-oculography (EOG) 2006. Doc. Ophthalmol. 113, 205-212 (2006).

35. S. Hillaire, A. Lécuyer, R. Cozot, G. Casiez, Using an eye-tracking system to improve camera motions and depth-of-field blur effects in virtual environments. 2008 IEEE Virtual Real. Conf., 47-50 (2008).

36. S. Lee, F. M. Li, A. Nathan, Influence of surface energy and roughness on hole mobility in solution-processed hybrid organic thin film transistors, IEEE J. Electron Devices Soc.6, 653657 (2018).

37. S. Lee, S. Jeon, A. Nathan, Modeling sub-threshold current-voltage characteristics in thin film transistors. J. Disp. Technol. 9, 883-889 (2013).

38. W. L. Kalb, S. Haas, C. Krellner, T. Mathis, B. Batlogg, Trap density of states in smallmolecule organic semiconductors: A quantitative comparison of thin-film transistors with single crystals. Phys. Rev. B. 81, 155315 (2010).

39. W. L. Kalb, B. Batlogg, Calculating the trap density of states in organic field-effect transistors from experiment: A comparison of different methods. Phys. Rev. B. 81, 035327 (2010).

40. S. Lee, A. Nathan, Conduction Threshold in Accumulation-Mode InGaZnO Thin Film Transistors. Sci. Rep. 6, 1-9 (2016).

41. R. A. Street, Localized states in doped amorphous silicon. J. Non. Cryst. Solids. 77-78, 1-16 (1985).

42. S. Lee, A. Nathan, Localized tail state distribution in amorphous oxide transistors deduced from low temperature measurements. Appl. Phys. Lett. 101 (2012).

43. K. P. Ghatak, S. Bhattacharya, Debye Screening Length: Effects of Nanostructured Materials (Springer, 2014).

44. M. Shur, M. Hack, Physics of amorphous silicon based alloy field-effect transistors. J. Appl. Phys. 55, 3831-3842 (1984).

45. T. Tiedje, J. M. Cebulka, D. L. Morel, B. Abeles, Evidence for Exponential Band Tails in Amorphous Silicon Hydride. Phys. Rev. Lett. 46, 1425-1428 (1980).

46. C. Liu et al., A unified understanding of charge transport in organic semiconductors: The importance of attenuated delocalization for the carriers. Mater. Horizons. 4, 608-618 (2017).

47. F. A. Padovani, R. Stratton, Field and thermionic-field emission in Schottky barriers. Solid State Electron. 9, 695-707 (1966).

48. A. S. Dahiya et al., Single-crystalline ZnO sheet Source-Gated Transistors. Sci. Rep. 6, 19232 (2016). 
49. F. N. Hooge, T. G. M. Kleinpenning, L. K. J. Vandamme, Experimental studies on 1/f noise. Reports Prog. Phys. 44, 479-532 (1981).

50. X. Cheng, S. Lee, A. Nathan, Deep Subthreshold TFT Operation and Design Window for Analog Gain Stages. IEEE J. Electron Devices Soc. 6, 195-200 (2018).

51. Y. Nara, M. Matsumara, An amorphous silicon integrated inverter. IEEE Trans. Electron Devices. 29, 1646-1649 (1982).

52. G. B. Raupp et al., Low-temperature amorphous-silicon backplane technology development for flexible displays in a manufacturing pilot-line environment. J. Soc. Inf. Disp. 15, 445-454 (2007).

53. S. D. Brotherton et al., High-speed, short-channel polycrystalline silicon thin-film transistors. Appl. Phys. Lett. 84, 293-295 (2004).

54. C. Lin, L. Cheng, Y. Lu, Y. Lee, H. Cheng, High-Performance Low-Temperature Poly-Si TFTs Crystallized by Excimer Laser Irradiation with Recessed-Channel Structure. IEEE Electron Device Lett. 22, 269-271 (2001).

55. B. Zhang, Z. Meng, S. Zhao, M. Wong, H.-S. Kwok, Polysilicon Thin-Film Transistors With Uniform and Reliable Performance Using Solution-Based. IEEE Trans. Electron Devices. 54, 1244-1248 (2007).

56. H. Hosono, in Handbook of Visual Display Technology, (Springer, 2012), 729-749.

57. R. Hayashi et al., Circuits using uniform TFTs based on amorphous In-Ga-Zn-O TFTs on Si substrates. J. Soc. Inf. Disp. 15, 915-921 (2007).

58. H. Klauk, Organic thin-film transistors. Chem. Soc. Rev. 39, 2643 (2010).

59. K. Fukuda et al., Fully-printed high-performance organic thin-film transistors and circuitry on one-micron-thick polymer films. Nat. Commun. 5, 4147 (2014).

60. L. Feng et al., All-solution-processed low-voltage organic thin-film transistor inverter on plastic substrate. IEEE Trans. Electron Devices. 61, 1175-1180 (2014).

61. J. Li, Z. Sun, F. Yan, Solution processable low-voltage organic thin film transistors with high-k relaxor ferroelectric polymer as gate insulator. Adv. Mater. 24, 88-93 (2012).

Acknowledgments: Funding: We thank EPSRC under Project EP/M013650/1, and EU under Projects DOMINO 645760, 1D-NEON 685758 and BET-EU 692373 for the generous support. C.J. acknowledges doctorate scholarship support from the China Scholarship Council (CSC) and the Cambridge Commonwealth, European and International Trust, and fellowship support from the IEEE Electron Device Society. Author contributions: C.J., H.W.C., X.C., H.M., and A.N. conceived of and designed the overall experiments. C.J. and H.W.C. carried out experiments and collected related data. C.J. was the only human subject for the eye tracking study, and H.W.C. recorded the experiment. X.C. contributed to circuit simulation and theoretical analysis. C.J., H.W.C., and A.N. initiated the study. C.J., H.W.C., X.C., H.M., D.H. and A.N. analyzed all the data and co-wrote the paper. All authors discussed the results and commented on the manuscript. 
Competing interests: The authors declare no competing interests. Data and materials availability: All data are available in the main text or the supplementary materials.

\section{Supplementary Materials:}

Materials and Methods

Supplementary Text

Figures S1-S12

Tables S1-S2

Movie S1

References (36-61)
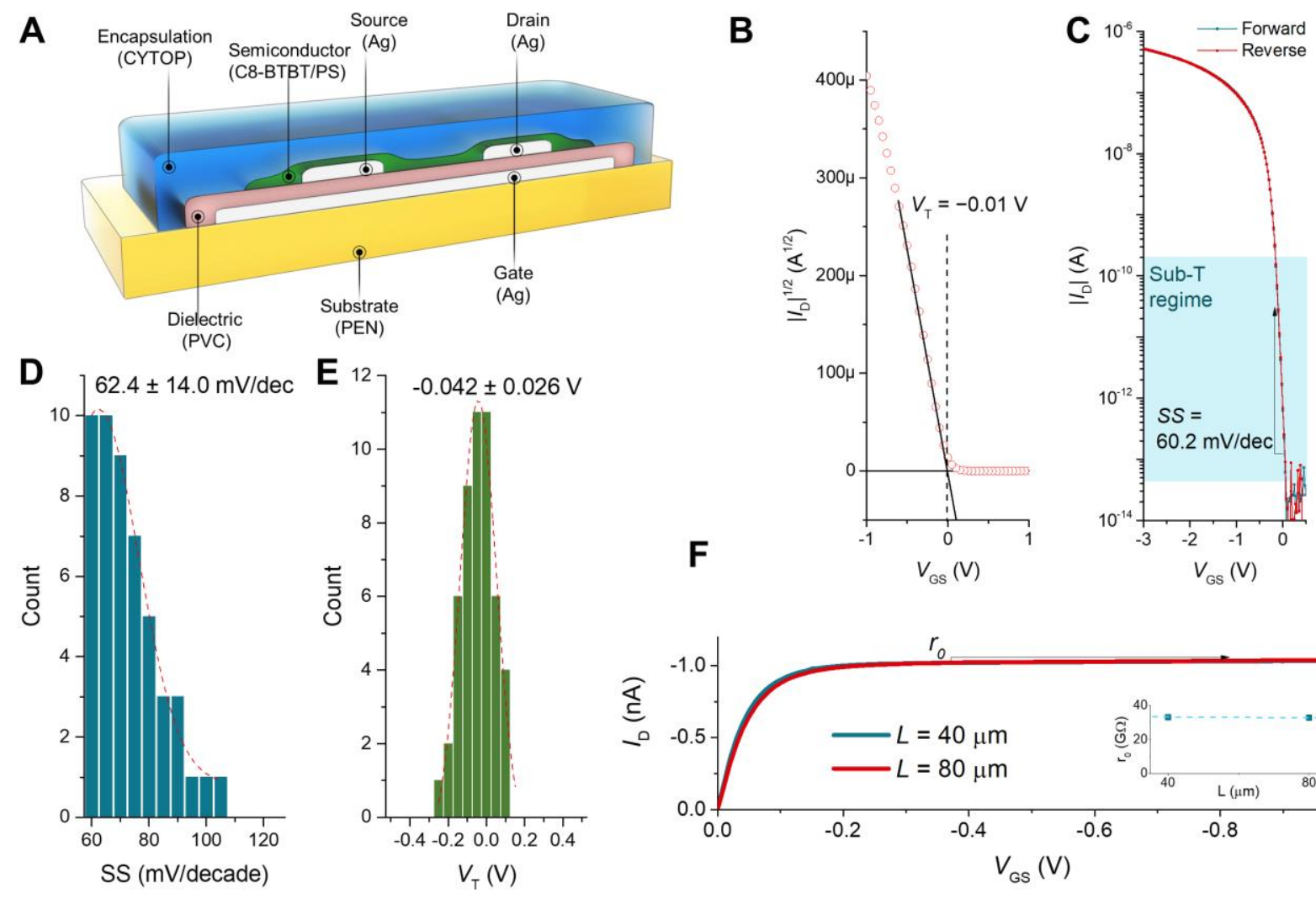

Fig. 1. Device structure and electrical characteristics. (A) Schematic cross section of the SBOTFT. Measured transfer characteristics ( $I_{\mathrm{D}}$ vs. $\left.V_{\mathrm{GS}}\right)$ of a typical device $(\mathbf{B})$ on a linear-scale, indicating the threshold voltage $\left(V_{\mathrm{T}}\right)$, and $(\mathbf{C})$ on a log-scale, indicating the subthreshold slope $(S S)$. Statistical distributions of (D) $S S$ and (E) $V_{\mathrm{T}}$ for 50 devices. The dashed lines indicate normal distributions. (F) Measured output characteristics ( $I_{\mathrm{D}}$ vs. $\left.V_{\mathrm{DS}}\right)$ indicating the output 
resistance $\left(r_{\mathrm{o}}\right)$ of devices with different channel length $(L)$ showing a full overlap of the characteristics. Inset shows $r_{0}$ vs. $L$.
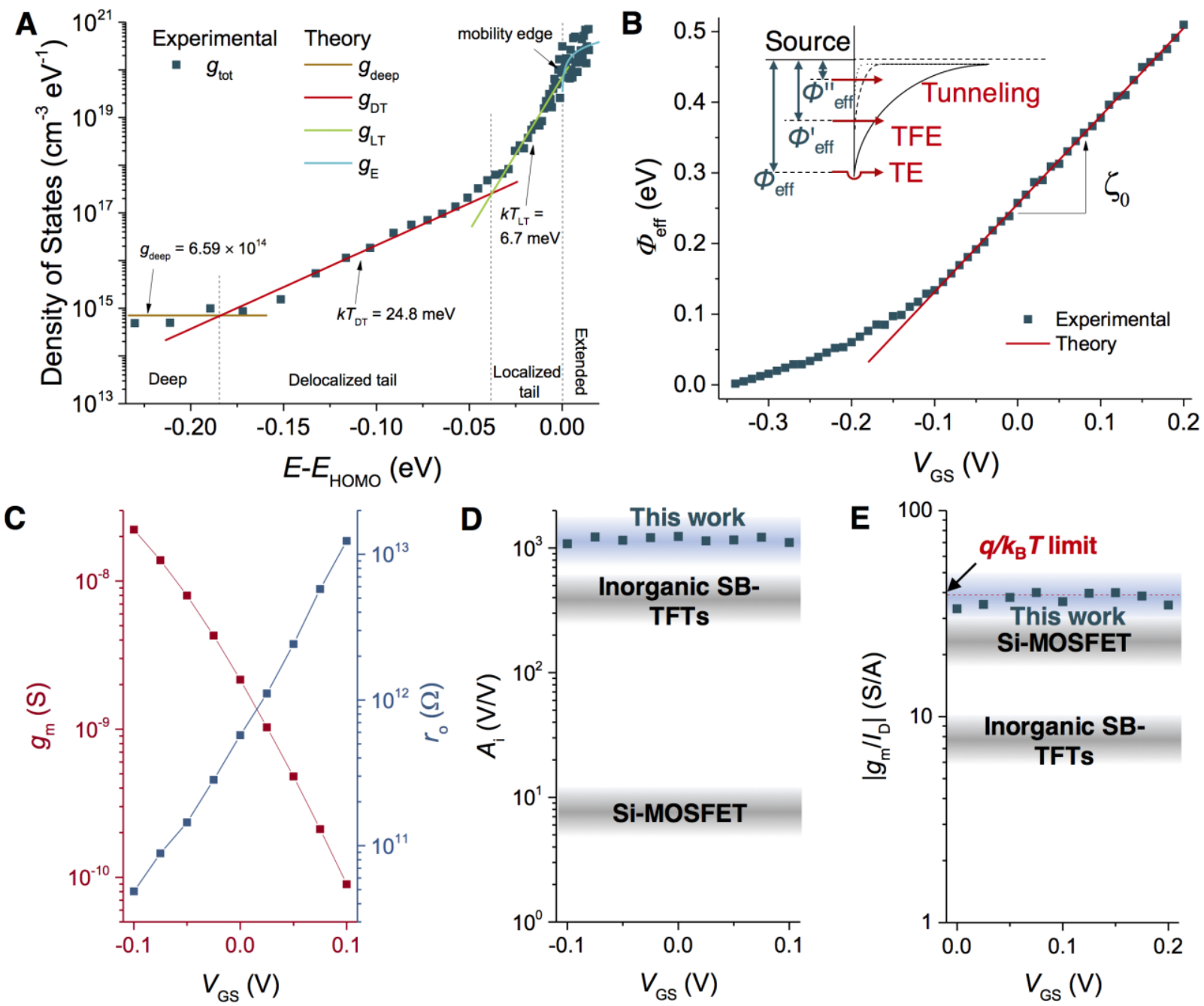

Fig. 2. Static parameters. (A) Density of states (DOS) for a typical device, indicating four different regimes: deep states, delocalized tail (DT) states, localized tail (LT) states, and extended states. The slopes in the DT and LT regimes indicate the characteristic energies $\left(k_{\mathrm{B}} T_{\mathrm{DT}}\right.$ and $k_{\mathrm{B}} T_{\mathrm{LT}}$, respectively). (B) Effective Schottky-barrier heights ( $\left.\Phi_{\mathrm{eff}}\right)$ as a function of $V_{\mathrm{GS}}$, indicating the gate-modulation factor $\left(\zeta_{0}\right)$ for the $\Phi_{\text {eff }}$ lowering (inset: schematic energy band diagram showing variation in effective $\Phi_{\text {eff }}$ and different charge carrier injection processes). (C) Experimental values for $g_{\mathrm{m}}$ and $r_{\mathrm{o}}$ as a function of $V_{\mathrm{GS}}$. (D) Measured intrinsic gain $\left(A_{\mathrm{i}}\right)$ as a 
function of $V_{\mathrm{GS}}$. (E) Experimental values of transconductance efficiency $\left(g_{\mathrm{m}} / I_{\mathrm{D}}\right)$ as a function of $V_{\mathrm{GS}}$ reaching the theoretical thermionic limit of $38.7 \mathrm{~S} / \mathrm{A}$.
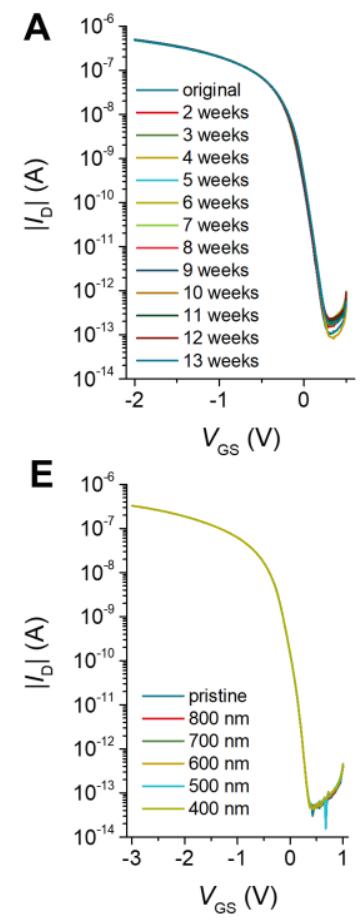
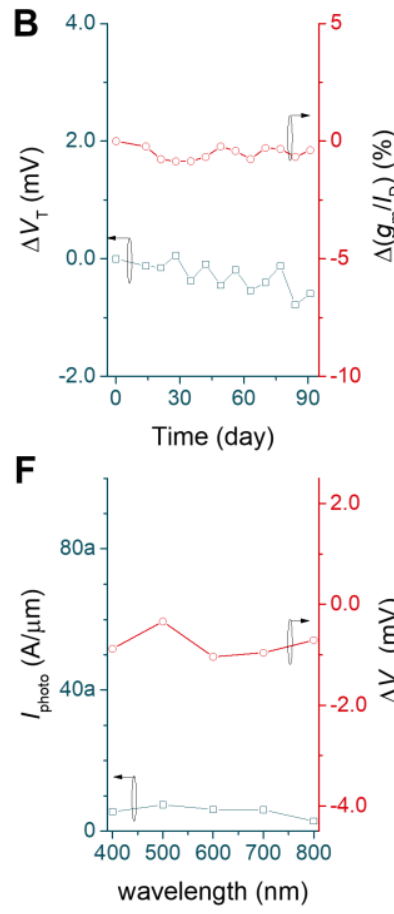

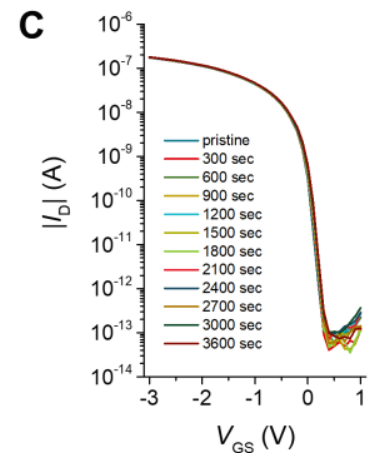

G

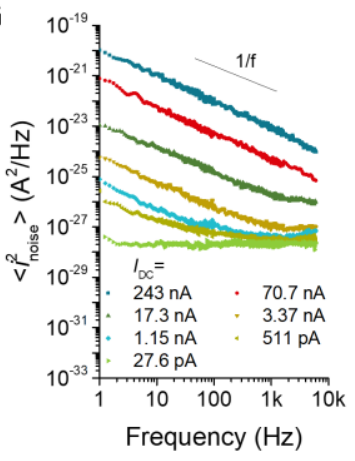

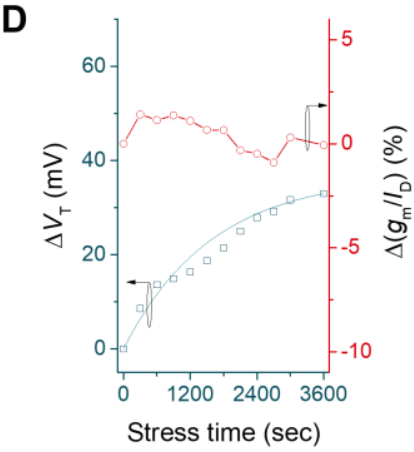

H

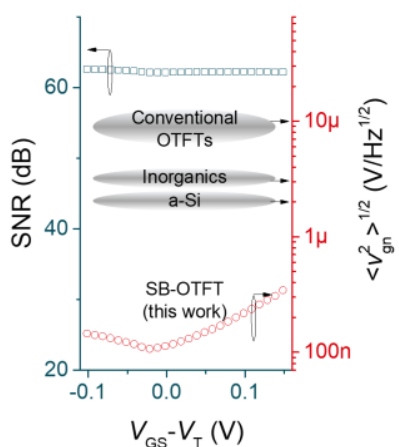

Fig. 3. Stability and reliability. (A) Measured transfer characteristics for storage under ambient conditions for the times indicated and $(\mathbf{B})$ change in absolute threshold voltage $\left(\Delta V_{\mathrm{T}}\right)$ and change in relative transconductance efficiency $\left[\Delta\left(g_{\mathrm{m}} / I_{\mathrm{D}}\right)\right]$ as a function of time. $(\mathbf{C})$ Measured transfer characteristics under negative bias stress $\left(V_{\mathrm{GS}}=V_{\mathrm{DS}}=-3 \mathrm{~V}\right)$ for the stress time indicated, and (D) $\Delta V_{\mathrm{T}}$ and $\Delta\left(g_{\mathrm{m}} / I_{\mathrm{D}}\right)$ as a function of stress time. (E) Measured transfer characteristics under light exposure, $(\mathbf{F})$ photocurrent $\left(I_{\text {photo }}\right.$ in $\left.\mathrm{A} / \mu \mathrm{m}\right)$ and $\Delta V_{\mathrm{T}}$ for different wavelengths $(400 \sim 800$ $\mathrm{nm})$. (G) Measured SB-OTFT current noise under different direct current biases (IDC). (H) 
Signal-to-noise ratio (SNR) in the near-threshold and subthreshold regimes, and input-referred voltage noise density at $100 \mathrm{~Hz}$.
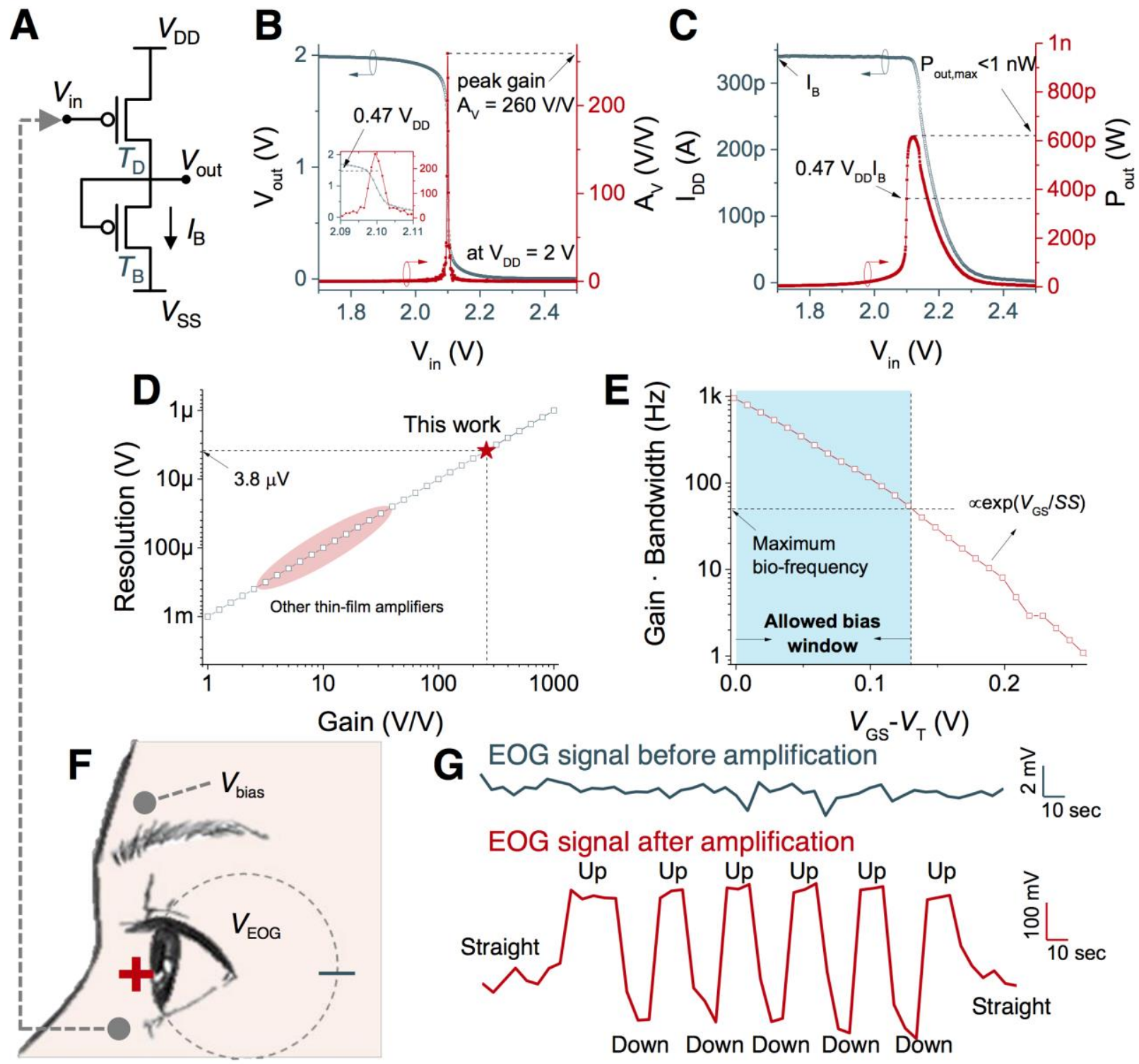

G

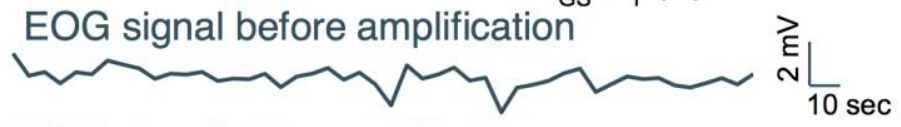

EOG signal after amplification

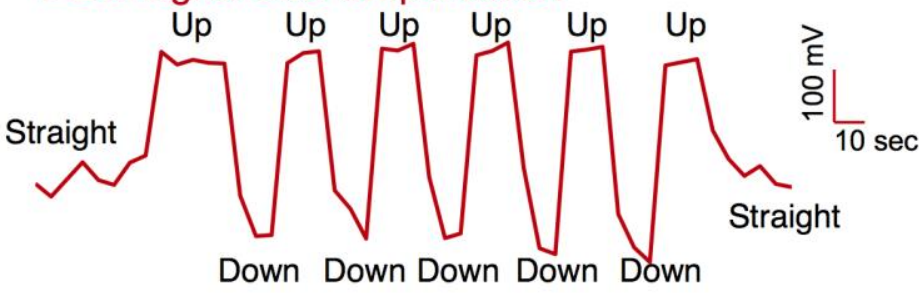

Fig. 4. Amplifier characteristics and demonstration of electro-oculography detection. (A)

Schematic circuit diagram of a common-source amplifier. (B) Measured output voltage $\left(V_{\text {out }}\right)$ and gain $\left(A_{\mathrm{V}}\right)$ as a function of input voltage $\left(V_{\mathrm{in}}\right)$. $(\mathbf{C})$ Measured operating current $\left(I_{\mathrm{DD}}\right)$ and power $\left(P_{\text {out }}\right)$ as functions of $V_{\text {in. }}$ (D) Resolution of electrophysiological signal detection as a function of gain. (E) Gain-bandwidth product as a function of $V_{\mathrm{GS}}$ in the subthreshold regime. (F) Operating principle and circuit configuration for electro-oculography (EOG) amplification with the amplifier. (G) EOG signal obtained before and after amplification. 